\title{
Magnetic and spectroscopic properties of supergranular-scale coronal jets and erupting loops in a polar coronal hole ${ }^{\star}$
}

\author{
J.-S. He ${ }^{1}$, E. Marsch ${ }^{1}$, W. Curdt ${ }^{1}$, H. Tian² ${ }^{2}$ C.-Y. Tu ${ }^{2}$, L.-D. Xia ${ }^{1,3}$, and S. Kamio ${ }^{1}$ \\ 1 Max-Planck-Institut für Sonnensystemforschung, Katlenburg-Lindau 37191, Germany \\ e-mail: jshept@gmail.com \\ 2 School of Earth and Space Sciences, Peking University, Beijing 100871, PR China \\ 3 School of Space Science and Physics, Shandong University, Weihai 264209, PR China
}

Received 1 April 2010 / Accepted 25 June 2010

\begin{abstract}
Context. Coronal jets and mass ejections associated with erupting loops are two distinct and frequently observed types of transient upflows of plasma in coronal holes (CHs). But the magnetic and spectroscopic properties of these events at the supergranular scale are not well known.

Aims. Here we aim at studying in a polar hole the plasma and field characteristics of coronal jets and erupting loops of a supergranular size, for which we use observations from XRT, EIS and SOT on Hinode as well as EUVI on STEREO.

Methods. The open magnetic field structures related to the coronal jets are obtained by magnetic field extrapolation into the corona from SOT magnetograms. Furthermore, we use the EIS observations to analyze ultraviolet line intensities and Doppler shifts in association with the erupting loops.

Results. We find that the coronal jet plasma is indeed ejected along open field lines, thus confirming the conjecture of jet formation in an open magnetic environment. The magnetic evolution at the jet base is investigated, and the results indicate that the interaction between two flux tubes of opposite magnetic polarities as well as the squeezing of several tubes with identical polarities might be responsible for the jet initiation. We reveal for the first time the spectroscopic signatures of a supergranular-size erupting loop at its early stage, which consists of three steps. The first step is the onset, which is featured by a sudden brightening of one footpoint, as well as by the occurrence of blueshifts along almost its entire path. The second step is the initial expansion of the closed loop, which is estimated to move upward at a speed of about $20 \mathrm{~km} \mathrm{~s}^{-1}$, as derived from the line-of-sight (LOS) blueshift and the loop enlargement projected onto the plane of the sky. In the third step, the loop's bright footpoint is apparently diminishing its intensity and enhancing its blueshift, which indicates that plasma upflow from the leg is filling the expanding loop volume.

Conclusions. From our results we conclude that in polar CHs, where the steady fast solar wind is known to emanate, there are also at least two possible ways of causing transient plasma outflows at supergranular scale. One is related to coronal jets guided by open field lines, the other to the eruption of closed loops, which is triggered by magnetic reconnection at their footpoints.
\end{abstract}

Key words. solar wind - Sun: corona - Sun: magnetic topology

\section{Introduction}

Polar coronal holes (CHs) are known to be regions on the Sun with large-scale open magnetic fields. The underlying highresolution vector-magnetic-field landscape in the polar photosphere has recently been reported by Tsuneta et al. (2008), on the basis of detailed observations from the spectro-polarimeter fed by the Solar Optical Telescope (SOT) onboard Hinode. It is now well understood that the polar $\mathrm{CH}$ is a major source region of the steady fast solar wind, as originally suggested by Krieger et al. (1973). The continuous outflow of the nascent fast solar wind was found to be channelled by coronal funnels, which stem from the network junctions in the chromosphere (Hassler et al. 1999; Tu et al. 2005b; Tian et al. 2010). However, coronal jets and mass ejections associated with erupting loops are also frequently observed transient upflows of plasma in $\mathrm{CHs}$, which may contribute to the solar wind as well. But their magnetic and spectroscopic properties at the supergranular scale are not well known. Here we aim at providing such new knowledge.

$\star 3$ movies (for Figs. 2-4) are only available in electronic form at http://www . aanda.org
Marsch et al. (2006) studied the structure of the solar transition region (TR) in a polar $\mathrm{CH}$ and found that it is not thermally stratified but strongly nonuniform and highly magnetically structured. Being anchored in the TR, coronal plumes are scattered all over the polar $\mathrm{CH}$, but their contribution to the fast solar wind still remains a matter of debate (e.g., Teriaca et al. 2003; Curdt et al. 2008). The spicules generally extend farther out in the polar $\mathrm{CH}$ than in other regions of the Sun (e.g., Xia et al. 2005), and were recently found to be a carrier of upward-propagating Alfvén waves (De Pontieu et al. 2007; He et al. 2009).

Coronal jets are more prevalent in polar $\mathrm{CHs}$ than in quietSun regions or in active regions (ARs). The number of recorded coronal jet events has increased dramatically since the operation of the X-Ray Telescope (XRT) aboard Hinode started, compared with their occurrence derived from the Soft X-ray Telescope (SXT) aboard Yohkoh (Cirtain et al. 2007). Cirtain et al. (2007) further pointed out that coronal jets with higher or lower velocities may result from different formation mechanisms, with fast jets related to magnetic reconnections, and slow ones driven by magnetosonic shock waves. The velocity structure of an elongated coronal jet was derived by Kamio et al. (2007), who reported a blueshift of $30 \mathrm{~km} \mathrm{~s}^{-1}$ in the LOS direction of the 
coronal jet. The falling-back of part of the jet plasma was inferred by Culhane et al. (2007), using the post-jet enhancement in a number of the cooler coronal lines observed by the EUV Imaging Spectrometer (EIS) onboard Hinode.

Some coronal jets showed signatures of a helical structure, and in consequence revealed the untwist processes as seen in the stereoscopic data obtained by the Extreme UltraViolet Imager (EUVI) onboard the twin STEREO spacecraft (Patsourakos et al. 2008). A three-dimensional (3D) scenario involving magnetic reconnection was suggested to explain the observed untwisting jet (Filippov et al. 2009; Pariat et al. 2009). However, to our knowledge the true magnetic-field structure underlying a coronal jet has hardly been reconstructed. Recently, Shimojo \& Tsuneta (2009) found that the emergence of patches of minority polarity in the polar $\mathrm{CH}$ may be responsible for coronal jets, and also for the coronal bright points found there.

Loop eruption is another type of coronal transient event, in which the plasma confined by a closed magnetic loop is seen to move outward, thus causing the loop to open partially at last, and then the plasma to escape from the Sun and perhaps to provide mass to the solar wind. One process of this kind is well known, namely the spectacular loop eruption leading to a largescale coronal mass ejection (CME). The speed of this CME can be as low as $30 \mathrm{~km} \mathrm{~s}^{-1}$ at their initial rising phase below $2 R_{\odot}$ (Gallagher et al. 2003), but usually reaches more than $400 \mathrm{~km} \mathrm{~s}^{-1}$ at $r=2 R_{\odot}$ (Sheeley et al. 1999). Some relatively small-scale eruptions of coronal plasmas accompanied by small filament eruptions (dark clouds) and propagating wave-like disturbance were coined mini-CMEs, which occur at the junctions of supergranular cells and appear to be activated by converging and rotating supergranular flows (Innes et al. 2009). Yet other small-scale loop eruptions do not show morphology components similar to those in CMEs.

Although the morphology of erupting loops, as well as their motions in the plane of the sky, have been intensively studied by using the evolution of the loop emission pattern, we are still short of information about the LOS motion of erupting loops. A strong outflow component was revealed in the dimming region of an AR during the eruption with the help of EIS observations (Jin et al. 2009). However, the signature of Doppler shifts along the entire erupting loop remained unclear. Nevertheless, that there is lasting coronal convection (guided by the strong coronal magnetic field) of the plasma in a stationary large closed-loop system of an AR was established by Marsch et al. (2008), who exploited the observed correlation between the maps of the stream-like Doppler shifts and of the extrapolated magnetic field. To identify the Doppler shifts associated with an erupting smaller-scale closed loop is one of the main objectives of our study.

In this paper, we analyse the magnetic and spectroscopic features of coronal jets and erupting closed loops, which are of supergranular size. First, we reconstruct the magnetic field structure resembling the emission pattern of coronal jet, and then we present the magnetic activity at the jet base in order to find out the possible physical driver for the jet. Subsequently, we show the evolution of the intensity and Doppler-flow over the initial period of the loop. The results will help us to better understand the initiation process of a supergranular-scale loop eruption at high solar latitude.

\section{Observations and data reduction}

The data sets we use here were obtained from a joint campaign of Hinode, the Solar Ultraviolet Measurements of Emitted
Radiation (SUMER), and the Transition Region and Corona Explorer (TRACE) between 15:00 and 21:00 UT on April 24, 2009, when all the instruments were targeted at the southern polar $\mathrm{CH}$. High cadence $(1 \mathrm{~min})$ observations from XRT with an Al-poly filter show the evolution of the emission pattern of coronal jets and erupting loops. Stokes $V$-magnetograms from the Narrow-band Filter Imager (NFI) of SOT with a cadence of about 6 min reveal the magnetic field activities at the base of the coronal jets. The temporal variation of the spectroscopic features of the coronal jets and erupting loops was observed by EIS, which repetitively rastered the same solar region with a repetition period of about 6 min. There are 13 raster positions in one cycle, and the exposure time is $25 \mathrm{~s}$ at every raster position. The slit with a width of $2^{\prime \prime}$ is adopted. Seven emission lines are recorded, Fe XII (186.74 A), Ca XVII (192.82 Å), Fe XII (195.12 ̊), Fe XVII (254.87 $)$, He II (256.32 $\AA$ ), Si X $(261.04 \AA)$, and Fe XV (284.16 $)$. Only Fe XII (195.12 $\AA$ ) and He II (256.32 $\AA$ ) lines have sufficient count rates in our case due to short exposure time and weak $\mathrm{CH}$ radiation. But the $\mathrm{He}$ II (256.32 $\AA$ ) line is blended by other lines, e.g., Si x(256.37 $\AA$ ), at the red wing near the solar limb (Young et al. 2007), and thus cannot be used. The footprint of the SUMER slit drifted on the solar disk from the central meridian position to the eastern side, and thus covered a solar- $x$-coordinate range of about $350^{\prime \prime}$.

In a way similar to Tu et al. (2005b), we thus used the SUMER data to study the Doppler-shifts of the TR in the polar $\mathrm{CH}$. We confirmed the conclusions reached by Tu et al. (2005b), namely that Ne VIII emitting at a high TR-temperature appeared to be blueshifted in most parts of the polar $\mathrm{CH}$, a notion indicating the formation of the nascent solar wind in the high TR layer. TRACE took snapshots of the polar CH in Fe IX/X $171 \AA$ with a cadence of $75 \mathrm{~s}$, whilst TRACE also occasionally took chromospheric images of the polar $\mathrm{CH}$ in the spectral band around $1550 \AA$. In addition, the observations from EUVI/STEREO-A in the Fe XII line with a cadence of 10 min showed the coronal jet and the erupting loop from another perspective with a different viewing angle.

We applied the procedures in the "SolarSoftWare" (SSW) package for the various instruments and in this way initially calibrated these datasets. We rotated the solar image sequences of the same instrument to a common time, and then aligned these sequences by implementing the Fourier cross-correlation technique ("tr_get_disp.pro" in SSW). Afterwards, we co-aligned the images from different instruments as described below. The SOT photospheric images were co-aligned with the TRACE chromospheric images, and the TRACE coronal images were coaligned with the XRT coronal images, as well as with the rasterscanned images from EIS. In this way, all the images from the photosphere to the corona were spatially co-aligned. Note that since the geometrical distortion of the SOT images remains uncalibrated, we can only co-align sub-areas of them case by case.

To derive the Doppler-shift distribution of Fe XII from EIS, we calculated the central positions of the spectral line at every spatial pixel, and then set a rest wavelength. The spectral center was derived by using the centroid method following, e.g., Dammasch et al. (1999). Before calculating the map of spectral center distribution, we needed to remove the artificial effects of slit tilt and orbital variation on the wavelength vectors of the line profiles at every spatial pixel. We employed the SSW routine "eis_slit_tilt.pro" to remove the silt-tilt effect. The orbital variation of spectral center, which is caused by instrument thermal effect during the orbit, results in the temporal variation of the spectral center of the spectral profile summed up over the slit. In 
practice, we used the temporal variation of the spectral center of Fe XII, which is strong and clean, to eliminate the orbital variation effect on all lines. The rest wavelength of Fe XII is assumed to be the given by the spectral center averaged over the whole map. Finally, we obtained calibrated Doppler-shift maps of the Fe XII line.

We adopted the method proposed by Seehafer (1978) to obtain potential-field extrapolations of the coronal magnetic field from photospheric magnetograms. This method has been successfully applied for studying the coronal magnetic fields in various regions (e.g., Marsch et al. 2004; Wiegelmann et al. 2005; Tu et al. 2005a; He et al. 2007; Tian et al. 2009). A direct comparison between the extrapolated magnetic field lines and the EUV-emitting closed loops in an AR was made by Feng et al. (2007). But the magnetic structures reconstructed in these previous studies were mainly on areas of the solar disk far away from the solar limb. To reconstruct the coronal magnetic structure in the polar $\mathrm{CH}$ and further compare it directly with the emission pattern, we need firstly to get a top view of the magnetogram, by rotating the magnetogram map from the polar $\mathrm{CH}$ position to the disk center, and secondly to rotate the extrapolated magnetic field lines back to the polar $\mathrm{CH}$ position, for the purpose of a direct comparison with the emission feature seen there. We assumed the magnetic field vectors to be in the radial direction, before obtaining the top view of the magnetogram. We note that the magnetic field distribution obtained from NFI of SOT can be used for extrapolation, although the exact magnetic flux remains un-calibrated. An estimate of the accurate magnetic flux in polar $\mathrm{CH}$ regions is problematic, since it is difficult to convert Stokes$V$ images observed by NFI/SOT into an accurate magnetogram without reference to the magnetograms from other instruments, e.g., MDI. Until now, only the magnetic flux in active regions has been derived from NFI/SOT Stokes- $V$ images by comparing it to the MDI observations (Chae et al. 2007). Nevertheless, temporal relative variation of the magnetic flux, i.e., increase or decrease in magnitude, may still be indicated by the NFI/SOT data.

\section{Analysis results}

In Fig. 1, we show an overview of the polar $\mathrm{CH}$ observed by XRT. Coronal bright points (CBPs) appear to be scattered all around the polar $\mathrm{CH}$, while relatively larger closed loops are located in the quiet-Sun region outside the polar $\mathrm{CH}$. Jet-like structures at times extend suddenly above the CBPs in the polar $\mathrm{CH}$. The observation areas of EIS and SOT are outlined, respectively, by the green-solid and green-dashed rectangles in Fig. 1. For clear position reference, we added a plot of the Stonyhurst heliographic grid (Thompson 2006) (dotted blue lines) to the solar disk in Fig. 1, and to the other figures below. The longitudinal and latitudinal intervals of the Stonyhurst heliographic grid are both set to be $15^{\circ}$. In the following subsections we will study a number of these jet-like structures in details.

\subsection{Possible drivers of the coronal jets and their guidance by the open field lines}

The launch of the first case of a coronal jet and its related magnetic field is displayed in Fig. 2. Figures 2(a1-a6) show the coronal X-ray jet over a period of $20 \mathrm{~min}$ from 19:09:56 to $19: 29: 56 \mathrm{UT}$. We can see that the jet is bright in radiation intensity and shows a sharp edge against the dark background. The magnetic field evolutions at the jet base are shown

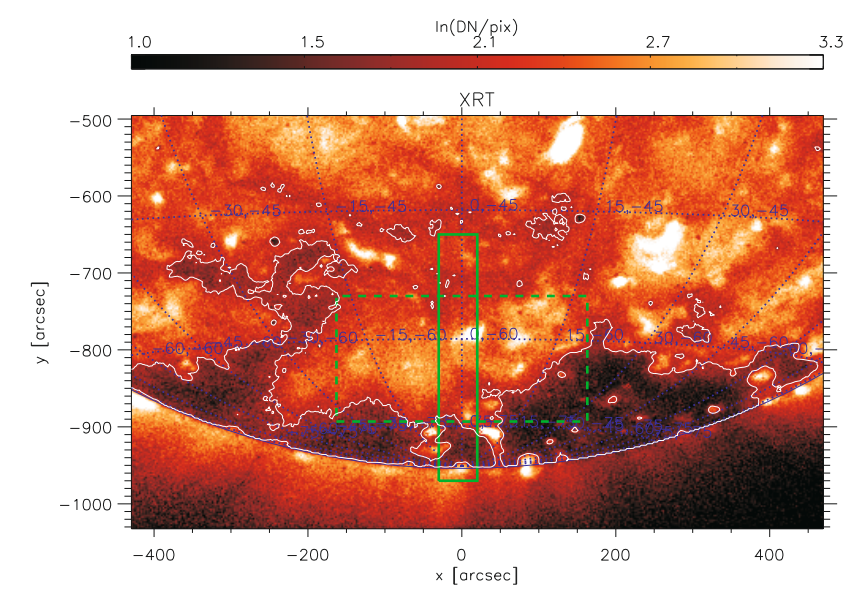

Fig. 1. XRT image of the southern polar $\mathrm{CH}$ at 17:57:35 UT. The green solid and dashed rectangles generally outline the observational areas of EIS and SOT, respectively. Blue dotted lines in this and other figures represent the Stonyhurst heliographic grids with both the longitudinal and latitudinal intervals being $15^{\circ}$. Blue solid arc lines in this and following figures denote the solar limb. Coronal hole boundaries are indicated by white contours.

in Figs. 2(b1-b3), in which the white and black colors represent the positive and negative $B_{\mathrm{LOS}}$. As marked by a yellow arrow in Fig. 2(b1), we can clearly see that during this period a group of three positive magnetic-field flux tubes was squeezed together by the supergranular flow. Based on this magnetic activity, we may attribute the ultimate driver of this coronal jet to the supergranular converging motions, which likely excited the shock wave propagating upward and raising the jet plasma. The shock wave has been suggested to be one of the causes for the jet launch (Cirtain et al. 2007). Magnetic reconnection may not be necessary for the jet launch in this case. If we adopt the un-calibrated Stokes- $V$ magnetogram to estimate the line-ofsight (LOS) magnetic flux, the positive magnetic flux in the blue rectangles of Figs. 2(b1-b3) is approximated to be $2.0 \times 10^{19}$, $1.9 \times 10^{19}$, and $1.9 \times 10^{19} \mathrm{Mx}$ at 19:09:32, 19:21:33, and 19:33:33, respectively, indicating little relative change. The negative magnetic flux, which is closely adjacent to the positive magnetic fields in the blue rectangles, is calculated to be $-2.5 \times 10^{18}$, $-3.5 \times 10^{18}$, and $-3.8 \times 10^{18} \mathrm{Mx}$, respectively. We note that this temporal variation of negative magnetic flux might be a LOS visual effect of the outward magnetic flux in the polar region, which may gradually incline away from the observer.

The associated magnetic field structure is presented in Figs. 2(c1, c2), with the blue lines representing the open field lines, which are able to reach up to $40 \mathrm{Mm}$. It is extrapolated from the magnetogram observed by SOT at 19:27 UT, which is the intermediate time between 19:21 UT (Fig. 2(b2)) and 19:33 UT (Fig 2(b3)). Figure 2(c1) shows the top view of the magnetic field structure, which exhibits a divergence pattern. Figure 2(c2) shows the magnetic structure as observed from the viewing angle of Hinode. It appears to consist of a bunch of field lines pointing uniformly toward the south of the solar disk, and permits a direct comparison with the ultraviolet emission pattern. We thus find a good consistency between the coronal jet and open field lines, both of which are directed toward the southwest when being projected onto the plane of the sky. We infer that the strong intensity and compact thickness of the coronal jet apparently results from the overlapping of field lines when they are viewed side-on from Hinode. 

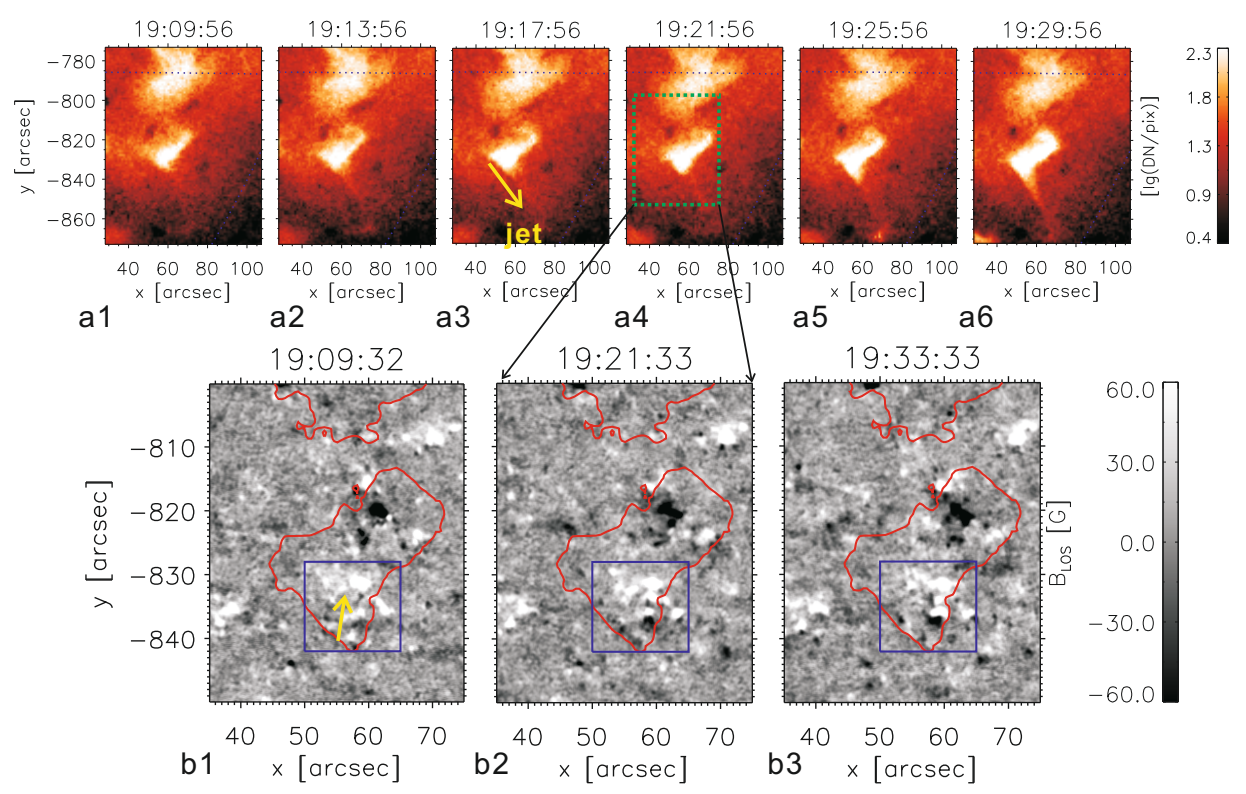

$\mathrm{b} 2^{40} \times\left[\begin{array}{ll}50 & 60 \\ & 70\end{array}\right.$
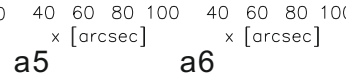

66

$19: 33: 33$
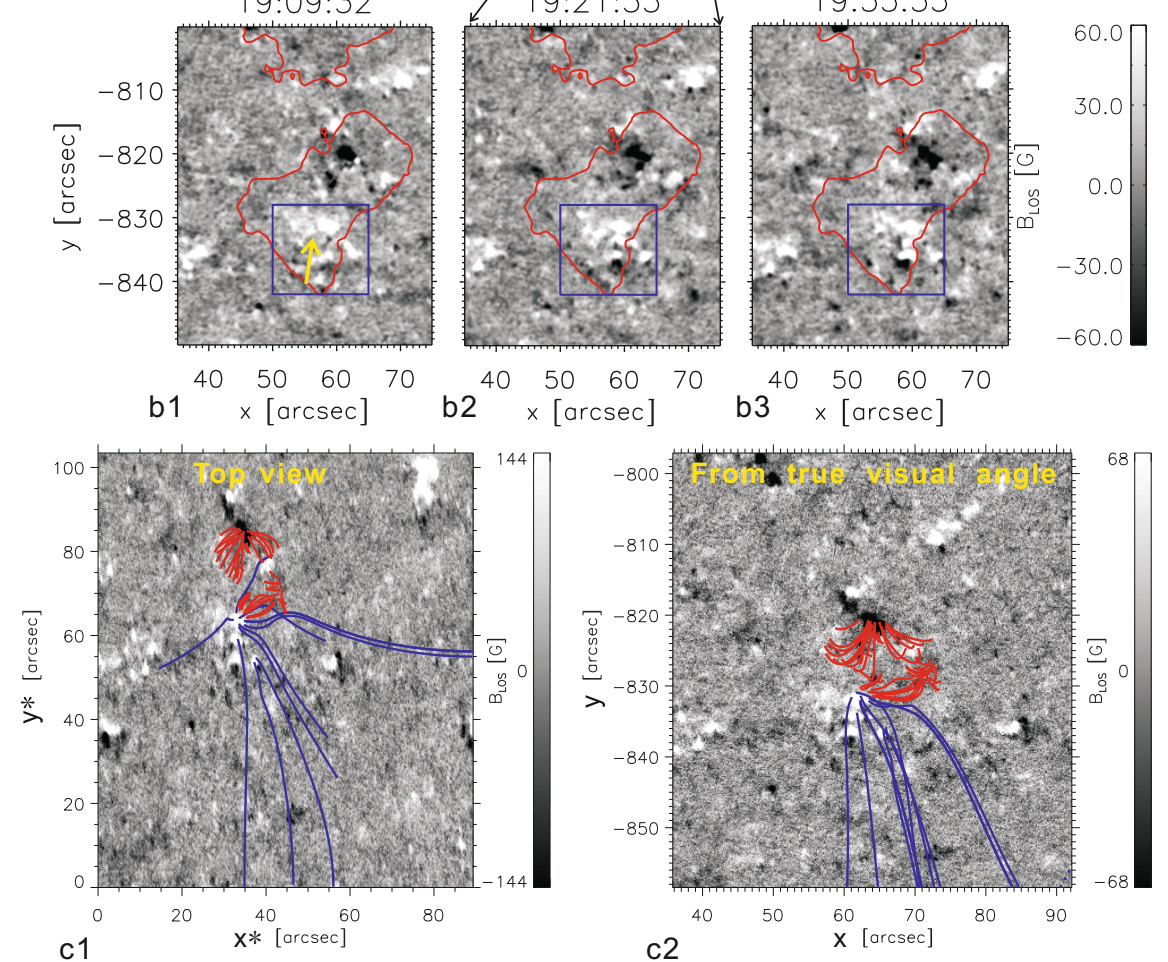

Fig. 2. a1-a6): Temporal variation of the first coronal jet observed from XRT over a period between 19:09:56 and 19:29:56. The yellow arrow indicates the direction of the jet. b1-b3): Corresponding magnetic field evolution at the jet base as observed from NFI of SOT. The yellow arrow marks a squeezing of three small patches of positive magnetic flux. The blue rectangles mark the area in which we estimate the relative change in magnetic flux. c1): Top view of the potential-field extrapolated magnetic structure related to the coronal jet at 19:27:32 UT. Blue lines denote the open field lines, which are reaching up to $40 \mathrm{Mm}$, while red lines represent the relatively low-lying field lines. c2): Side view of the same magnetic field lines as in c1) from the true visual angle of Hinode satellite. This side view of the magnetic structure from the true visual angle can be directly compared to the jet emission pattern shown in a1-a6). The temporal evolution in soft X-rays as observed with XRT/Hinode in the panels a1-a6) is shown in the movie "Figure2_XRT_movie.gif" available in the online version.

Figure 3 illustrates the second case of a coronal jet guided by open magnetic fields. The evolution of this X-ray jet from 15:18:59 to 15:34:59 UT is shown in Figs. 3(a1-a5). The magnetic field activities at its coronal base are shown in Figs. 3(b1-b3), from which we can identify that a small area of negative magnetic flux flushed into a large area of positive flux and then disappeared (the yellow arrow in Fig. 3(b1)). This phenomenon suggests that the cancellation of opposite polarities at the network junction might be the driver of this jet. To track the evolution of this small area of negative magnetic flux in detail, magnetograms with higher cadence are needed. Based on the un-calibrated Stokes- $V$ magnetograms, we approximate the negative magnetic flux in the blue rectangles of Figs. 3(b1-b3) to be $-9.4 \times 10^{17},-8.9 \times 10^{17}$, and $-7.1 \times 10^{17} \mathrm{Mx}$ at 15:16:31, $15: 28: 32$, and 15:40:32, respectively. This is a slight relative decrease in magnetic flux with negative polarity. On the other hand, the total flux of the neighboring positive magnetic fields, which dominate over the negative fields, is not significantly affected by the slight decrease in magnetic flux due to possible magnetic cancellation. The related magnetic field lines, which are viewed from the viewing angle of Hinode, are displayed in Fig 3(c), and demonstrate again that coronal jets are guided by the open field lines. We note that in this case the temporal variation of the small-scale reconnecting magnetic geometry cannot be easily described by using the force-free extrapolation. But the largescale reconfiguration of reconnecting magnetic geometry around a nano-flare in an AR has been demonstrated by using the forcefree extrapolation (He et al. 2010).

\subsection{Spectroscopic and stereoscopic features of a coronal jet at the south pole}

In Fig. 4, we show the launch of the third case of a coronal jet, which occurs at the south pole and is not covered by a SOT magnetogram. But we were lucky that this jet event was observed simultaneously by XRT, EIS, and EUVI/STEREO-A. Therefore, we could investigate it in both its spectroscopic and stereoscopic aspects. Figures 4(a1-a6) show the XRT observations. It can 


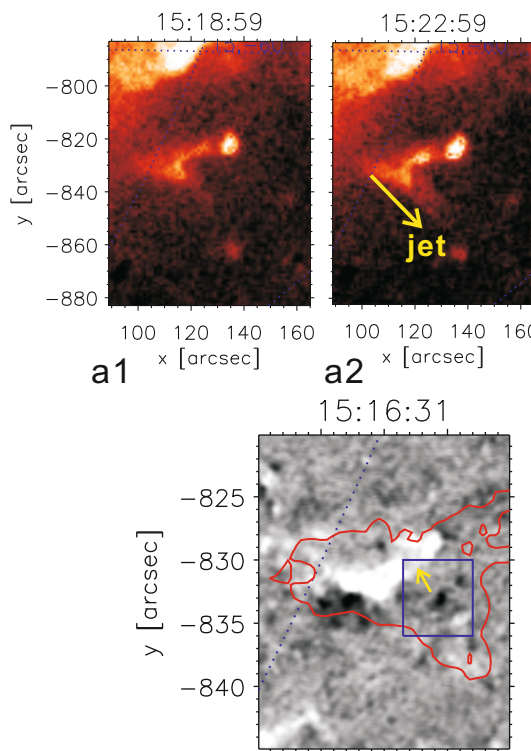

105110115 b1 $\times$ [arcsec $]$
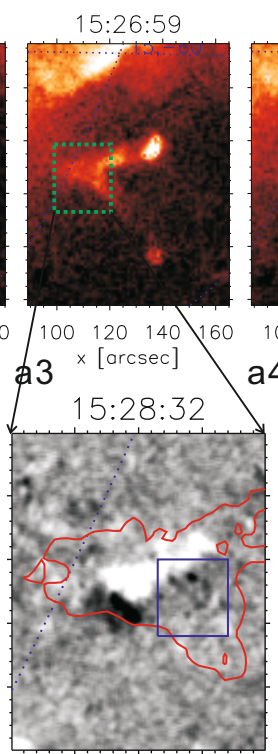

105110115

b2 $\times$ [arcsec $]$
$15: 30: 59$

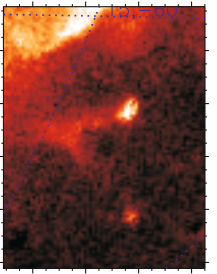

$\begin{array}{llllllll}100 & 120 & 140 & 160 & 100 & 120 & 140 & 160\end{array}$

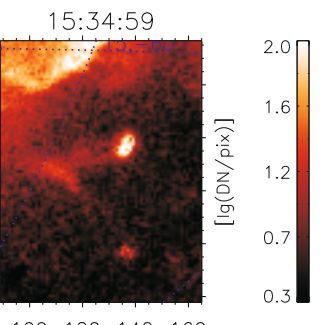

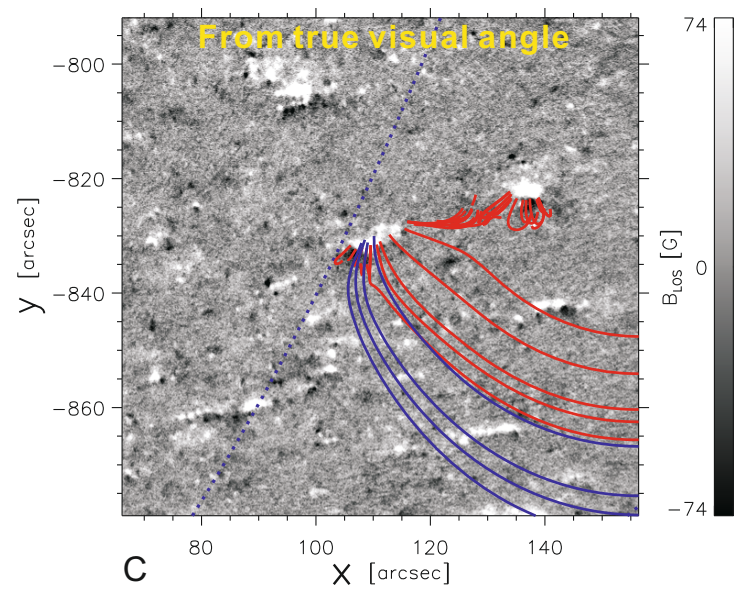

Fig. 3. a1-a5): Temporal evolution of the second coronal jet observed from XRT between 15:18:59 and 15:34:59 UT. The yellow arrow indicates the jet direction. b1-b3): Magnetic field activity at the jet base observed from NFI of SOT. The yellow arrow denotes the motion of a negative magnetic field element toward a large patch of positive magnetic flux. The blue rectangles denote the region, in which we estimate the relative change in magnetic flux. c): Side view of the magnetic field structure at 15:22:31 UT from the true viewing angle of Hinode. A movie for this soft X-ray jet event, "Figure3_XRT_movie.gif", is available in the online journal.

be seen from Fig. 4(a4) that this jet attains a " $Y$ " shape, with the head and tail of the " $Y$ " representing the already existent closed loop below, as well as the newly ejected jet above, respectively. Before the jet launch, a sudden brightening of one leg of the closed loop took place at some moment between 19:05 and 19:10 UT (Figs. 4(a1, a2)). This sudden brightening was observed by EIS and EUVI/STEREO-A in Fe XII $195 \AA$, too. We speculate that this flaring is likely caused by the magnetic reconnection between the open field and the closed loop, with one of its legs closely approaching the open field line. The closed loop may have already been existent before the reconnection, rather than having newly emerged from the subsurface.

The corresponding Doppler-shifts of the brightening foot of the closed loop are displayed in Figs. 4(b4) and (b6), which show an obvious blue-to-red shift pattern related to this bright point. This Doppler-shift distribution may indicate a siphon flow in the newly reconnected small-scale closed loop, or perhaps a bi-directional flow around the reconnection site (Tian et al. 2008). An alternative interpretation may be the rotational motion around a macro-spicule (Banerjee et al. 2000) in the closed loop. But we should keep in mind that this pattern was obtained by raster scans rather than simultaneous observations. The western foot of the " $Y$ "-shaped jet, which is concealed behind the solar limb in the XRT images, is revealed in the EUVI/STEREO-A image as observed from another viewing angle of about $47^{\circ}$ apart from the Hinode satellite. This coronal jet in the EUVI images also shows an apparent " $Y$ "-shaped structure at 19:15 and 19:25 UT (Figs. 4(c3, c4)).

\subsection{Spectroscopic observations of a supergranular-scale erupting loop}

In this subsection, we discuss the spectroscopic signature of an supergranular-scale erupting loop. The related magnetic closed 


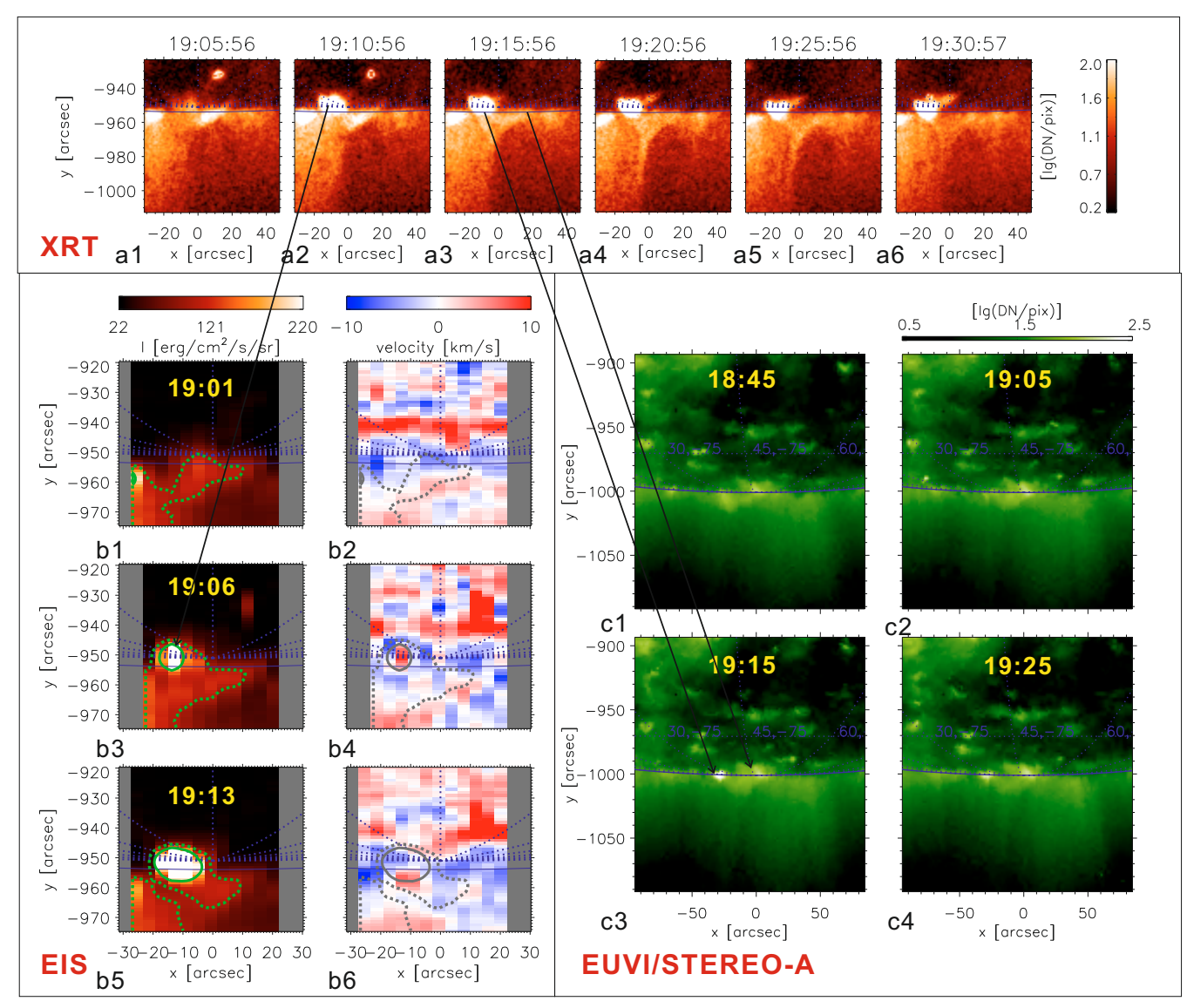

Fig. 4. a1-a6): Dynamic evolution of the third coronal jet occurring at the south pole observed from XRT between 19:05:56 and 19:35:57 UT. b1-b6): Temporal variation of the spectroscopic feature at three moments in time of the jet root obtained from EIS raster scanning. Two levels of strong radiation intensity are outlined by the green solid and dotted lines in the intensity images, and superposed on the Doppler-shift distributions with the gray solid and gray dotted lines. c1-c4): Observations of the coronal jet occurrence from EUVI/STEREO-A. The temporal evolution of the soft X-ray jet launch is displayed in the movie "Figure4_XRT_movie.gif" available in the online version.

loop and emission pattern are shown in Fig. 5. Figures 5(a1) and (a2) display the top view and side view of the extrapolated magnetic field structure, respectively. We can see that the span distance between two footpoints of the closed loop is about $60^{\prime \prime}$, which is about twice the size of a typical supergranule. Thus we call this a supergranular-scale closed loop. This erupting loop was neither accompanied by small-filament eruption nor wavelike disturbance at the front, therefore it is unlikely to be a miniCME like those in Innes et al. (2009). The side view of the closed magnetic loop from the viewing angle of Hinode is superimposed on the images of the X-ray intensity, the Fe XII intensity, and the Fe XII Doppler-shift (Figs. 5(b), (c1), and (c2)) for a direct comparison. We can see that the magnetic structure overall very well fits with the radiation structure. The small bright patch around ( $\left.x=20^{\prime \prime}, y=-780^{\prime \prime}\right)$ corresponds to the smallscale $\left(<30^{\prime \prime}\right)$ closed arcade. The bright eastern leg (starting from $\left.\left(x=-10^{\prime \prime}, y=-780^{\prime \prime}\right)\right)$ of the meso-scale closed emitting loop coincides with the dense eastern legs of the magnetic field line bunch. The scattered western legs of the meso-scale closed field lines are consistent with the diffuse emission pattern.

The details of the eruption process of this supergranularscale closed loop over the period between 19:00 and 22:00 UT are illustrated in Fig. 6. Figures 6(a1-a7) and (b1-b7) show the EIS observations of the temporal evolution of the Fe XII intensity and Doppler shift, respectively. XRT observations of the eruption process in soft X-ray are displayed in Figs. 6 (c1-c7). We find that the eruption process consists of three different steps: onset, initial expansion, and upflows from the footpoint. The eruption onset occurring at 20:23 UT is characterized by a flaring at the eastern footpoint and by a brightening extending along the eastern leg (Fig. 6(a2)). Meanwhile, there appears a blue-to-red shift at the eastern footpoint, and a blueshift along almost the entire path of the closed loop (Fig. 6(b2)). Before 20:00 UT, an obvious cancellation between opposite polarities was identified near the eastern foot from the SOT images. But as we are lacking the magnetic field at the time of eruption onset, we can only conjecture that the presumed magnetic reconnection at the eastern footpoint may be the onset trigger, which is similar to the conclusion by Chen \& Shibata (2000) that the magnetic reconnection at the edge of closed loops may trigger the CME onset. The initial upward expansion took place after the onset, showing a blueshift in the LOS direction along nearly the whole loop and a slow enlargement of the loop size projected onto the plane of the sky (Figs. 6(b3-b5)). The expansion speed component in the LOS direction is estimated to be $15 \mathrm{~km} \mathrm{~s}^{-1}$ at maximum, and the speed component in the plane of the sky is estimated to be $10 \mathrm{~km} \mathrm{~s}^{-1}$ at maximum. Therefore, this supergranular-scale loop expanded upward somewhat slowly with a speed of about $20 \mathrm{~km} \mathrm{~s}^{-1}$ during its initial expansion from 20:13 to 20:36 UT.

After the initial expansion, the emission pattern of the loop appears more diffuse, which is possibly caused by a relatively fast expansion after an initially slow expansion. Another obvious 

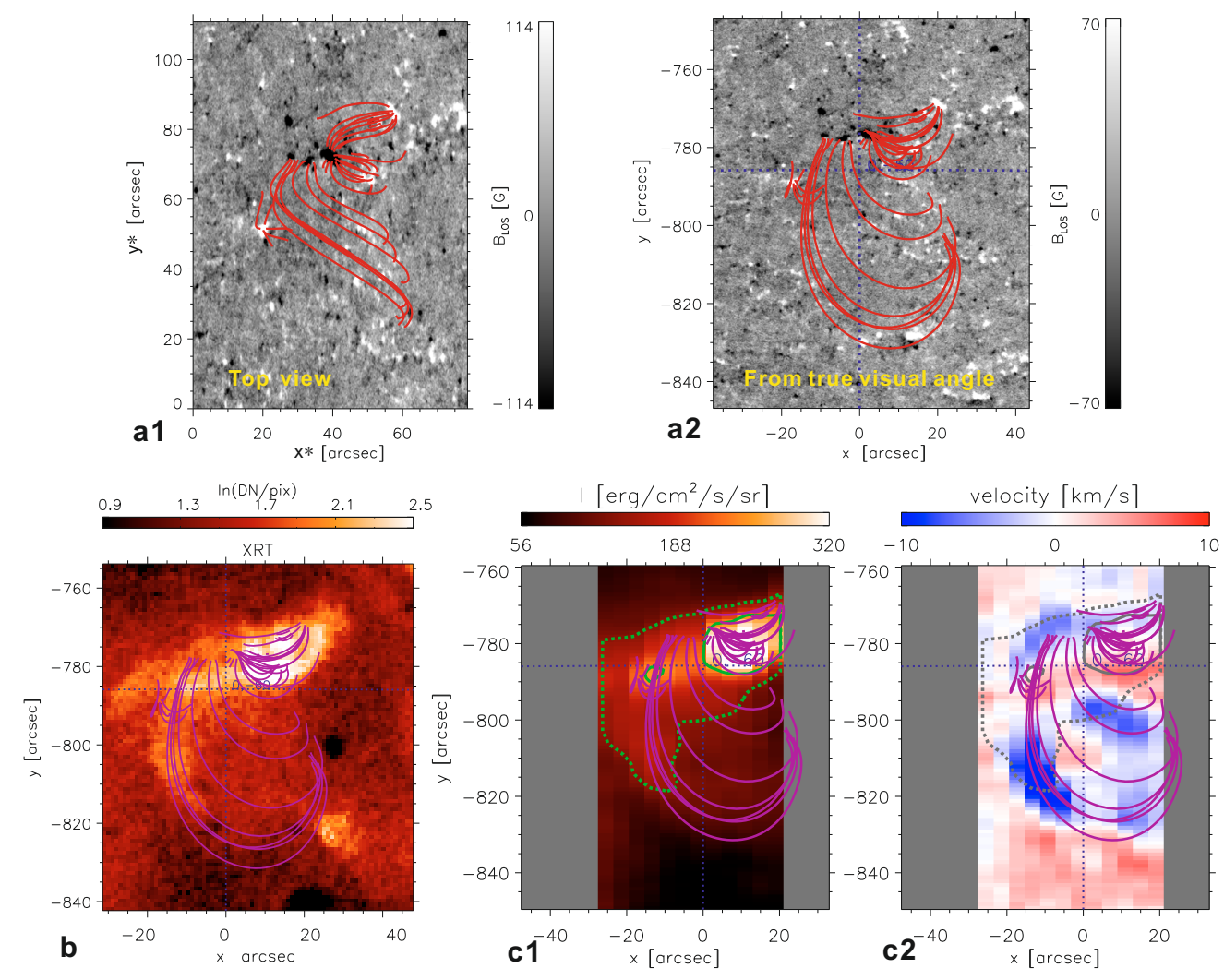

Fig. 5. a1): Top view of the closed magnetic loop at 19:33 UT related to the an erupting closed loop observed in soft X-ray; b) and Fe XII $195 \AA$ (c1). a2): Side view of this closed magnetic loop from the true viewing angle of Hinode. b): Soft X-ray image of the erupting closed loop at 20:23 UT. c1, c2): Intensity and Doppler-shift of the erupting closed loop observed by EIS in Fe XII at around 20:23 UT.

change after the initial expansion is the radiation intensity and Doppler shift at the eastern foot, which shows a dimming in intensity and a transition to blueshift (Figs. 6(a7, b7)). The dimming and blueshift at the eastern footpoint are manifestations that the plasma at the footpoint was flowing upward to fill in the expanded volume, which is similar to the flow pattern in the large-scale CMEs of previous studies (Jin et al. 2009). The upflow from the footpoint also has an obvious signature in soft $\mathrm{X}$-ray as observed by XRT. We note that this eruption is quite weak compared to mini-CMEs in the quiet Sun, and thus we do not find in a differential image sequence the outward propagation of a wave-like disturbance or the expansion of a dimming region. The further eruption stage of a typical CME, i.e., current sheet formation and magnetic reconnection below the rising flux rope, is not present in these limited observations.

\section{Summary and discussion}

We investigated in detail the supergranular-scale coronal jets and erupting loops in a southern polar $\mathrm{CH}$ region of the Sun on the basis of observations from XRT/Hinode, SOT/Hinode, EIS/Hinode, and EUVI/STEREO-A. The magnetic field evolutions at the base of the coronal jets were revealed. We suggest two possible driver mechanisms for the jet launch: one is the squeezing of magnetic fields with identical polarity, and the other is the interaction between magnetic fields with opposite polarities. We extrapolated the coronal magnetic field, rotated it to the viewing angle of the Hinode satellite, and compared it directly with the ultraviolet emission pattern in the image. We thus found that coronal jets are guided by open field lines, which supports previous speculations.
An X-ray (and EUV) emitting loop prone to eruption is found to be confined by (extrapolated) closed magnetic field lines. An unprecedentedly complete record of the spectroscopic features of the erupting closed loop was presented, using the repetitive raster-scanning images from EIS. There are three steps of the loop eruption. The first step is the onset, which is characterized by a sudden brightening at its eastern footpoint. This abrupt brightening suggests magnetic reconnection at the footpoint to be a possible mechanism triggering the onset. Associated with the brightening is the blueshift along nearly the entire closed loop except for its eastern footpoint. This blueshift indicates that the closed loop started to expand upwardly. Then the loop went into its second step of eruption after the onset, during which its shape was (indicated by the LOS blueshift on it) slowly enlarged, when looking at its projection onto the plane of the sky. The true expansion speed is estimated to be about $20 \mathrm{~km} \mathrm{~s}^{-1}$ during the $13 \mathrm{~min}$ initial slow expansion. At the third step, the emission pattern and blue shift of this closed loop disappeared, possibly due to rapid diffusion as the result of a fast expansion later on. Simultaneously, an obvious blueshift as well as intensity dimming appeared at its eastern footpoint, thus indicating an upflow of plasma from the foot into the expanding volume. We believe this study will shed new light on our understanding of the physical process of an eruption event in its initial phase, whatever the size scale may be.

Parts of the mass released from the coronal jets and erupting loops in the polar $\mathrm{CH}$ are considered to be a probably important seed mass for the solar wind emanating from the polar $\mathrm{CH}$. The highly dynamic nature of the polar $\mathrm{CH}$, which involves magnetic reconnection in the network as a crucial plasma driver, strongly suggests the necessity of incorporating 3-D magnetic 


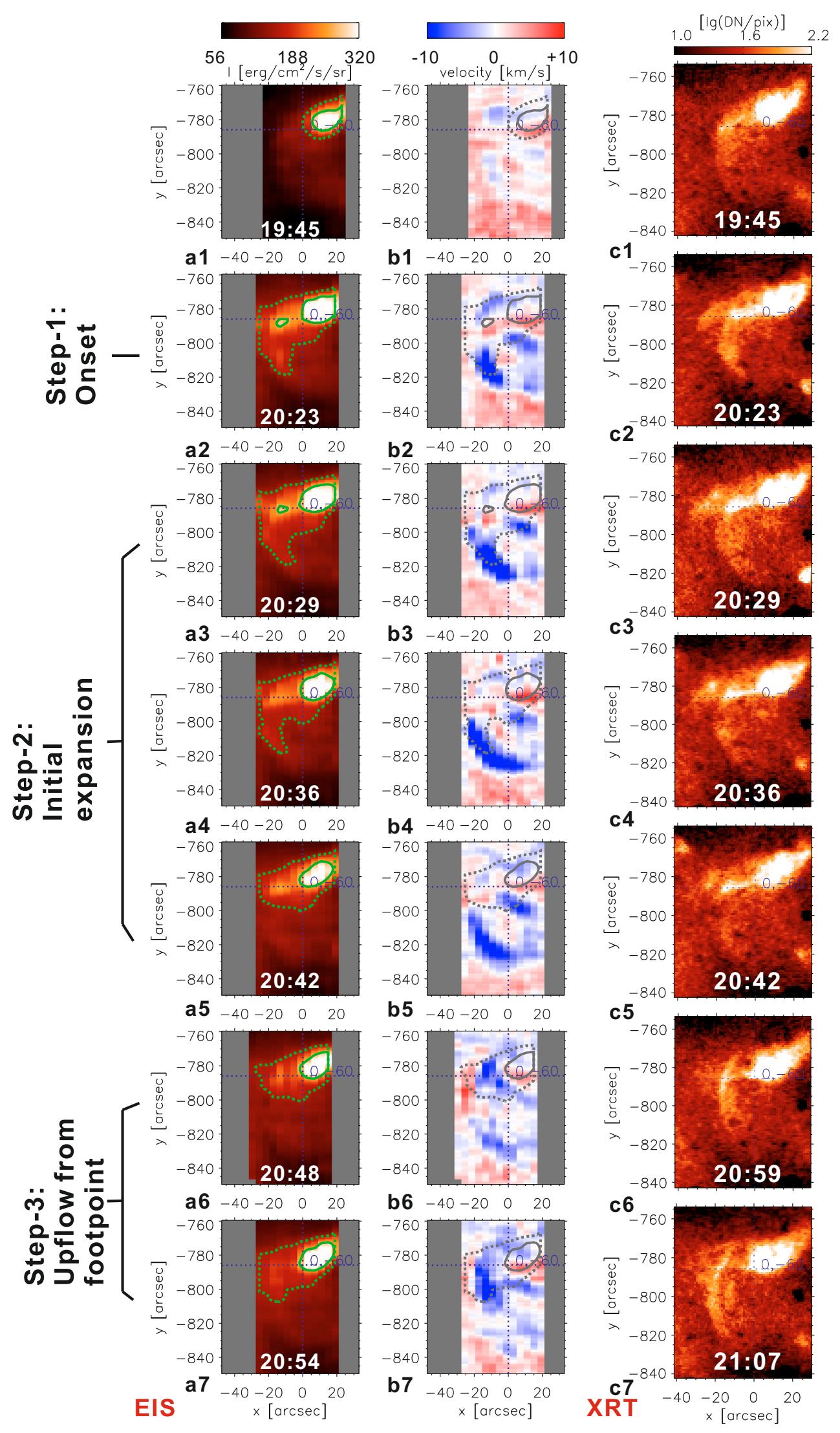

Fig. 6. a1-a7) and b1-b7): Dynamic evolution of the intensity and Doppler-shift of the erupting closed loop observed from EIS. Two levels of strong intensity contours are outlined respectively by the green solid and dotted lines in the intensity images, and superposed on the Doppler-shift images with the gray solid and gray dotted lines. Three steps of the eruption are indicated on the left side of the figures. c1-c7): Temporal variation of the erupting closed loop observed from XRT. 
reconnection into future modelling of the solar wind origin. Until now, the study of the dynamics in the polar $\mathrm{CH}$ is still insufficient, due to the lack of observations to be made from spacecraft out of the ecliptic. The "Solar Orbiter" mission (Marsch et al. 2005 ) is hoped to provide these key observations in the near future.

Acknowledgements. Hinode is a Japanese mission developed and launched by ISAS/JAXA, with NAOJ as domestic partner and NASA and STFC (UK) as international partners. It is operated by these agencies in co-operation with ESA and NSC (Norway). We acknowledge the teams from Hinode, SUMER and TRACE for their help to accomplish our proposal of this joint observation, and the team from EUVI/STEREO for making their data publically available on the internet. We thank the referee for his/her valuable comments. J.-S. He is supported by the post-doctoral fellowship at MPS. C.-Y. Tu and H. Tian are supported by the National Natural Science Foundation of China (NSFC) under Contract Nos. 40874090, 40931055, and 40890162. L.-D. Xia is supported by NSFC under Contract No. 40974105.

\section{References}

Banerjee, D., O'Shea, E., \& Doyle, J. G. 2000, A\&A, 355, 1152

Chae, J., Moon, Y., Park, Y., et al. 2007, PASJ, 59, 619

Chen, P. F., \& Shibata, K. 2000, ApJ, 545, 524

Cirtain, J. W., Golub, L., Lundquist, L., et al. 2007, Science, 318, 1580

Culhane, L., Harra, L. K., Baker, D., et al. 2007, PASJ, 59, 751

Curdt, W., Wilhelm, K., Feng, L., \& Kamio, S. 2008, A\&A, 481, L61

Dammasch, I. E., Wilhelm, K., Curdt, W., \& Hassler, D. M. 1999, A\&A, 346, 285

De Pontieu, B., McIntosh, S. W., Carlsson, M., et al. 2007, Science, 318, 1574

Feng, L., Inhester, B., Solanki, S. K., et al. 2007, ApJ, 671, L205

Filippov, B., Golub, L., \& Koutchmy, S. 2009, Sol. Phys., 254, 259
Gallagher, P. T., Lawrence, G. R., \& Dennis, B. R. 2003, ApJ, 588, L53 Hassler, D. M., Dammasch, I. E., Lemaire, P., et al. 1999, Science, 283, 810 He, J., Tu, C., \& Marsch, E. 2007, A\&A, 468, 307

He, J., Tu, C., Marsch, E., et al. 2009, A\&A, 497, 525

He, J., Marsch, E., Tu, C., Tian, H., \& Guo, L. 2010, A\&A, 510, A40

Innes, D. E., Genetelli, A., Attie, R., \& Potts, H. E. 2009, A\&A, 495, 319

Jin, M., Ding, M. D., Chen, P. F., Fang, C., \& Imada, S. 2009, ApJ, 702, 27

Kamio, S., Hara, H., Watanabe, T., et al. 2007, PASJ, 59, 757

Krieger, A. S., Timothy, A. F., \& Roelof, E. C. 1973, Sol. Phys., 29, 505

Marsch, E., Wiegelmann, T., \& Xia, L. D. 2004, A\&A, 428, 629

Marsch, E., Marsden, R., Harrison, R., Wimmer-Schweingruber, R., \& Fleck, B. 2005, Adv. Space Res., 36, 1360

Marsch, E., Zhou, G., He, J., \& Tu, C. 2006, A\&A, 457, 699

Marsch, E., Tian, H., Sun, J., Curdt, W., \& Wiegelmann, T. 2008, ApJ, 685, 1262

Pariat, E., Antiochos, S. K., \& DeVore, C. R. 2009, ApJ, 691, 61

Patsourakos, S., Pariat, E., Vourlidas, A., Antiochos, S. K., \& Wuelser, J. P. 2008, ApJ, 680, L73

Seehafer, N. 1978, Sol. Phys., 58, 215

Sheeley, N. R., Walters, J. H., Wang, Y., \& Howard, R. A. 1999, J. Geophys. Res., 104, 24739

Shimojo, M., \& Tsuneta, S. 2009, ApJ, 706, L145

Teriaca, L., Poletto, G., Romoli, M., \& Biesecker, D. A. 2003, ApJ, 588, 566

Thompson, W. T. 2006, A\&A, 449, 791

Tian, H., Curdt, W., Marsch, E., \& He, J. 2008, ApJ, 681, L121

Tian, H., Marsch, E., Curdt, W., \& He, J. 2009, ApJ, 704, 883

Tian, H., Tu, C., Marsch, E., He, J., \& Kamio, S. 2010, ApJ, 709, L88

Tsuneta, S., Ichimoto, K., Katsukawa, Y., et al. 2008, ApJ, 688, 1374

Tu, C., Zhou, C., Marsch, E., et al. 2005a, ApJ, 624, L133

Tu, C.-Y., Zhou, C., Marsch, E., et al. 2005b, Science, 308, 519

Wiegelmann, T., Xia, L. D., \& Marsch, E. 2005, A\&A, 432, L1

Xia, L. D., Popescu, M. D., Doyle, J. G., \& Giannikakis, J. 2005, A\&A, 438, 1115

Young, P. R., Del Zanna, G., Mason, H. E., et al. 2007, PASJ, 59, 857 